Pesq. Vet. Bras. 34(Supl.1):87-91, dezembro 2014 DOI: 10.1590/S0100-736X2014001300016

\title{
Avaliação da pressão arterial sistêmica em cães obesos: comparação entre os métodos oscilométrico e doppler ultrassônico ${ }^{1}$
}

\author{
Gláucia B. Pereira-Neto², Márcio A. Brunetto ${ }^{3 *}$, Tatiana Champion ${ }^{4}$, Edna M.G. \\ Ortiz $^{5}$, Aulus C. Carciofi ${ }^{5}$ e Aparecido A. Camacho ${ }^{5}$
}

\begin{abstract}
Pereira-Neto G.B., Brunetto M.A., Champion T., Ortiz E.M.G., Carciofi A.C. \& Camacho A.A. 2014. [Systemic blood pressure evaluation by oscillometric versus doppler ultrasonographic methods in obese dogs.] Avaliação da pressão arterial sistêmica em cães obesos: comparação entre os métodos oscilométrico e doppler ultrassônico. Pesquisa Veterinária Brasileira 34(Supl.1):87-91. Hospital Veterinário, Faculdade de Agronomia e Medicina Veterinária, Universidade de Brasília, Campus Universitário Darcy Ribeiro, Asa Norte, Brasília, DF 70910-900, Brazil. E-mail: mabrunetto@usp.br

Obesity is the most common nutritional problem in dogs and it can cause various harmful effects on animal health. However, the effect of this condition on systolic blood pressure (SBP) in obese dogs is controversial. The indirect method of measuring PAS is the most commonly used in veterinary medicine for the diagnosis of systemic hypertension, because it is more practical and easy to access. There is little scientific information about the comparison of the two non-invasive methods of measurement of blood pressure in obese dogs. Therefore, the objective of this study was to evaluate the SBP in obese dogs by comparing both indirect methods of measuring blood pressure, with oscillometric and doppler vascular in order to verify the differences in blood pressure values, but also the best method to assess the SBP dogs in this body score condition. The study complain blood pressure measurement of 50 dogs, with were divided in obese dogs with mean body condition score (BCS) of $8.42 \pm 0.50(n=25)$ and dogs with ideal BCS of $4.56 \pm 0.51(n=25)$. At comparison of blood pressure values, SBP values obtained by doppler method $(152 \pm 16 \mathrm{mmHg})$ were higher than the oscillometric $(136 \pm 11 \mathrm{mmHg})$. Correspondence analysis determined by multivariate statistical analysis showed correlation between body condition and the procedure of the SBP. These findings suggest that the indirect method doppler can better reflect the SBP in obese dogs.
\end{abstract}

INDEX TERMS: Obesity, canine, hypertension, indirect method.

RESUMO.- A obesidade é o problema nutricional que mais acomete os cães e pode causar vários efeitos deletérios a saúde animal. No entanto, o efeito desta condição sobre a pressão arterial sistólica (PAS) em cães obesos é controverso. Os métodos indiretos de mensuração da PAS são os

\footnotetext{
${ }^{1}$ Recebido em 19 de setembro de 2014.

Aceito para publicação em 6 de janeiro de 2015.

${ }^{2}$ Departamento de Medicina Veterinária, Faculdade de Agronomia e Medicina Veterinária, Universidade de Brasília (UnB), Campus Universitário Darcy Ribeiro, Asa Norte, Brasília, DF 70910-900, Brasil. E-mail: glauciabpn@unb.br

${ }^{3}$ Departamento de Nutrição e Produção Animal, Faculdade de Medicina Veterinária e Zootecnia, Universidade de São Paulo (USP), Campus de Pirassununga, Av. Duque de Caxias Norte 170, Pirassununga, SP 13635-900, Brasil. *Autor para correspondência: mabrunetto@usp.br
}

mais comumente utilizados na medicina veterinária para o diagnóstico da hipertensão sistêmica, por serem mais práticos e de fácil acesso. Não foram localizados na literatura estudos que tenham comparado os dois métodos para esta avaliação em cães obesos. Diante do exposto, o objetivo

\footnotetext{
${ }^{4}$ Departamento de Medicina Veterinária, Faculdade de Medicina Veterinária, Universidade Federal da Fronteira Sul, Rua Edmundo Gaievski 1000, Cx. Postal 253, Realeza, PR 85770-000, Brasil. E-mail: tatiana.champion@uffs.edu.br

${ }^{5}$ Departamento de Clínica e Cirurgia Veterinária, Faculdade de Ciências Agrárias e Veterinárias (FCAV), Universidade Estadual Paulista Júlio de Mesquita Filho (Unesp), Campus de Jaboticabal, Via de Acesso Prof. Paulo Donato Castellane s/n, Jaboticabal, SP 14884-900, Brasil. E-mails: ednacardio@hotmail.com, aulus.carciofi@gmail.com, camacho@fcav. unesp.br
} 
desse estudo foi avaliar a PAS em cães obesos, comparando os métodos indiretos de mensuração da pressão arterial, oscilométrico e doppler ultrassônico, a fim de verificar as diferenças nos valores obtidos, como também o método mais indicado para aferir a PAS em cães nesta condição. Foram avaliados 50 cães, incluindo 25 obesos com escore de condição corporal (ECC) médio de 8,42 $\pm 0,50$ e 25 cães com peso corporal ideal com ECC médio de 4,56 $\pm 0,51$. Os resultados revelaram valores superiores da PAS obtidos pelo método doppler $(152 \pm 16 \mathrm{mmHg})$ comparados ao oscilométrico $(136 \pm 11 \mathrm{mmHg})$. A análise de correspondência determinada por análise estatística multivariada demonstrou maior correspondência entre a condição corporal e o método de avaliação da PAS. Esses achados sugerem que o método indireto doppler pode aferir com mais precisão os valores de PAS em cães obesos.

TERMOS DE INDEXAÇÃO: Obesidade, canino, hipertensão, método indireto.

\section{INTRODUÇÃO}

Vários estudos já foram realizados para avaliar as técnicas de mensuração da pressão arterial em cães, como a comparação dos métodos diretos e indiretos em animais sedados, anestesiados e conscientes (Bodey et al. 1994, Bodey et al. 1996, Meurs et al. 1996). No entanto, desconhece-se estudos comparativos entre os métodos indiretos para a mensuração da pressão arterial em cães obesos, como também qual o melhor método indicado para detecção da hipertensão arterial.

A obesidade é o problema nutricional que mais acomete cães na atualidade, os quais são considerados clinicamente obesos quando o peso corporal excede o ideal para o seu tamanho corporal em pelo menos 15\% (Laflamme 2001). Esta afecção está associada a várias alterações clínicas e metabólicas, como resistência à insulina, problemas articulares, aumento de mediadores inflamatórios circulantes, pancreatite e redução da expectativa de vida (Laflamme 2006). Entretanto, a relação entre o excesso de peso corporal e o aumento da pressão sanguínea em cães obesos ainda não está totalmente esclarecida. Estudos experimentais que avaliaram a pressão arterial sistólica (PAS) pelo método oscilométrico em cães obesos observaram discreto aumento, inferior a $5 \mathrm{mmHg}$, quando comparado a cães com peso corporal ideal (Bodey et al. 1996), mas essa alteração não foi observada ao mensurar a PAS pelo doppler ultrassônico (Remillard et al. 1991).

De acordo com o consenso estabelecido pelo American College of Veterinary Internal Medicine (ACVIM) (Brown et al. 2007), a hipertensão arterial sistêmica é diagnosticada quando os valores de pressão arterial sistólica encontram-se superiores a $150 \mathrm{mmHg}$ e superiores a $95 \mathrm{mmHg}$ para a pressão arterial diastólica. No entanto, deve-se sempre considerar as pequenas variações que podem atingir diferenças de aproximadamente $10 \mathrm{mmHg}$, relacionadas a idade, sexo, escore de condição corporal, raça e interferências externas, como a síndrome do jaleco branco, que pode ocorrer durante a mensuração da pressão arterial em cães. Os métodos confiáveis e consistentes de mensuração da pressão arterial sistêmica são essenciais para a confirmação do diagnóstico de hipertensão sistêmica, além disso, essa confirmação deve ser feita por mensurações repetidas. Diversos métodos podem ser utilizados para aferir a pressão sanguínea, no entanto, os métodos não-invasivos são mais simples e de fácil realização na prática clínica (Brown \& Henik 1998, Carr 2001).

No método oscilométrico, o manguito de oclusão do fluxo é insuflado até atingir pressão acima da sistólica e em seguida desinsuflado lentamente por aumentos de 5 a $10 \mathrm{mmHg}$, enquanto o microprocessador mensura e realiza a média das amplitudes resultantes da oscilação da pressão sanguínea exercida sobre a parede da artéria. A eficácia na utilização do método oscilométrico pode ficar prejudicada em animais muito pequenos (Brown \& Henik 1998, Carr 2001). Já o método doppler ultrassônico baseia-se nas alterações da frequência entre o som emitido e os ecos que retornam (resultante do movimento das células sanguíneas ou da parede dos vasos) para detectar o fluxo sanguíneo em uma artéria superficial. Essa alteração na frequência, o desvio doppler, é convertida em sinal audível. Esse método é adequado principalmente para animais de pequeno porte. Quando se insufla o manguito aderido ao esfingomanômetro, o fluxo sanguíneo se oclui com a primeira pressão acima da sistólica e os sons não são mais audíveis. À medida que o manguito é desinsuflado de forma lenta, o movimento de retorno das células sanguíneas ou da parede arterial produz sinais de fluxo característico durante a sístole (Carr 2001).

0 objetivo desse estudo foi avaliar a pressão arterial sistólica em cães obesos e com peso corporal ideal, através da comparação dos métodos indiretos de mensuração da pressão arterial sistêmica, oscilométrico e doppler ultrassônico, a fim de verificar se existe diferença entre os mesmos, como também a metodologia mais indicada para aferir a pressão arterial sistólica em cães obesos, uma vez que não se sabe se o excesso de tecido adiposo localizado ao redor das regiões de mensuração, como membros e cauda, pode interferir na detecção de valores confiáveis.

\section{MATERIAL E MÉTODOS}

Animais. Foi realizado um estudo prospectivo observacional com 50 cães adultos, entre machos e fêmeas e de diferentes raças, sendo vinte e cinco obesos e vinte e cinco com escore de condição corporal (ECC) ideal. Os cães foram admitidos como obesos ao apresentarem ECC maior ou igual a oito, e os com peso corporal ideal (controle) com ECC entre quatro e cinco. Todos os animais foram provenientes da rotina de atendimento dos Serviços de Clínica Médica e Nutrição Clínica de Cães e Gatos e do Laboratório de Pesquisa em Nutrição e Doenças Nutricionais de Cães e Gatos de um hospital veterinário universitário estadual.

Antes do início do estudo, efetuou-se triagem composta por exame físico, hemogramas e dosagem sérica de alanina amino transferase, fosfatase alcalina, creatinina, uréia, proteina total, albumina, colesterol, triglicerídeos e glicose em jejum, assim como exame eletrocardiográfico e ecocardiográfico e dosagens hormonais para exclusão de endocrinopatias. Somente participaram do estudo indivíduos com resultados dentro do intervalo de referência para os parâmetros físicos, bioquímicos e cardíacos. Portanto, foram aceitos somente animais obesos por ingestão excessiva de alimento, excluindo-se as demais causas de obesidade.

Este estudo esteve de acordo com os princípios éticos de experimentação animal, adotado pelo Colégio Brasileiro de Experi- 
mentação Animal (COBEA) e foi aprovado pela Comissão de Ética e Bem Estar Animal (CEBEA) da Faculdade de Ciências Agrárias e Veterinárias da Universidade Estadual Paulista, com protocolo número 001785-09.

Diagnóstico da obesidade. 0 diagnóstico da obesidade foi realizado por meio da inspeção e palpação diretas e baseado no escore de condição corporal de acordo com o sistema de nove pontos descrito por Laflamme et al. (1997). Foram considerados obesos os animais que apresentaram ECC $\geq 8$, ou seja, costelas não palpáveis debaixo de grande quantidade de gordura subcutânea, depósitos de gordura visíveis na região lombar e na base da cauda, cintura muito pouco aparente ou não visível e curvatura abdominal ausente. A avaliação deste parâmetro foi realizada sempre pelos dois primeiros autores deste estudo, experientes nessa classificação.

Mensuração indireta da pressão arterial sistêmica. A pressão arterial sistólica foi mensurada utilizando-se primeiro o doppler de pressão ultrassônico ${ }^{6}$ e em seguida o aparelho de pressão oscilométrico ${ }^{7}$ de acordo com técnicas previamente descritas (Brown et al. 2007). Para tanto, os animais foram colocados em ambulatório tranquilo, para que se familiarizassem ao ambiente durante quinze minutos antes da mensuração. Em ambos os métodos, os cães foram gentilmente contidos em decúbito lateral direito, e na sequência o manguito foi acoplado sempre na posição distal do úmero esquerdo. A circunferência do membro anterior esquerdo de cada animal foi mensurada para determinação do comprimento e a largura ideal do manguito (largura $=40 \%$ da circunferência do membro). No método doppler ultrassônico utilizou-se a artéria superficial palmar do membro anterior esquerdo para a detecção audível do pulso.

Em cada avaliação obtiveram-se cinco tomadas consecutivas da PAS. 0 primeiro valor obtido em ambos os métodos foi descartado. Desta forma, o valor final foi representado pela média aritmética das três aferições que apresentaram valores mais aproximados (Brown et al. 2007).

Análise estatística. Os valores médios da PAS obtidos nos dois grupos pelos diferentes métodos, oscilométrico e doppler ultrassônico, após serem avaliados quanto ao padrão de normalidade pelo teste Anderson - Darling a 1\%, foram comparados pelo teste de análise de variância (ANOVA) seguido pelo teste de Tukey. A análise estatística foi realizada em nível de significância de 5\%, utilizando o programa SAS System (Schlotzhauer \& Littel 1997). Adicionalmente empregou-se a análise de correspondência múltipla com o objetivo de explorar as possíveis correspondências entre os valores de PAS, método de aferição da PAS estudados e condição corporal. Para esta análise, utilizou-se o software STATISTICA $^{8}$, segundo metodologia descrita por Hair et al. (2006).

\section{RESULTADOS}

Os cães obesos apresentaram ECC médio de $8,42 \pm 0,50$, sendo 20 fêmeas e cinco machos, de diferentes raças, incluindo beagle $(n=16)$, basset $(n=1)$, cocker spaniel $(n=1)$, daschund $(n=1)$, sheep dog $(n=1)$ e sem raça definida $(n=5) .0$ grupo controle foi constituído de cães sem raça definida, com ECC médio de 4,56 $\pm 0,51$, clinicamente saudáveis, 14 machos e 11 fêmeas.

A análise estatística dos valores da PAS desse estudo demonstrou padrão de distribuição normal. Desta forma, a média, o desvio-padrão e o intervalo de confiança confe-

\footnotetext{
${ }^{6}$ Ultrasonic Doppler Flow Detector - Model 811-BTS, Parks Medical Electronics, Inc.

${ }^{7}$ DX 2710 - DIXTAL Pressão não-invasiva.

${ }^{8}$ STATISTICA (Data analysis software system), STATSOFT, Inc. (2004), OK, USA.
}

Quadro 1. Valores médios \pm desvios-padrão da pressão arterial sistêmica sistólica mensurada por dois métodos em cães obesos $(n=25)$ e em cães com escore corporal ideal (controle, $n=25$ )

PAS Oscil controle PAS Doppler controle PAS Oscil obesos PAS Doppler obesos

$137,27 \pm 9,90 \mathrm{a} \quad 145,09 \pm 15,81 \mathrm{ab} \quad 136,52 \pm 11,00 \mathrm{a} \quad 152,24 \pm 16,15 \mathrm{~b}$

PAS = pressão arterial sistêmica sistólica, Oscil = método oscilométrico, Doppler = método Doppler.

Médias \pm desvios-padrão seguidas por letras minúsculas diferentes indicam diferença significativa a 0,05\%.

riram referência aceitável para a determinação da pressão sistêmica em ambos os grupos. A mensuração da pressão arterial sistólica foi realizada em ambos os métodos, segundo o protocolo estabelecido pelas diretrizes do consenso do ACVIM para identificação, avaliação e manejo da hipertensão sistêmica em cães e gatos (Brown et al. 2007), procurando evitar os fatores que pudessem resultar no falso diagnóstico de hipertensão.

Em cães obesos, a média da pressão arterial sistêmica sistólica obtida pelo método doppler foi superior a obtida pelo método oscilométrico ( $\mathrm{p}=0,0001)$. Os cães com ECC ideal não apresentaram diferenças na comparação das médias dos valores obtidos por ambos métodos indiretos (Quadro 1).

Apesar da diferença entre os métodos detectada no grupo dos animais obesos, não houve diferença significativa nos valores da PAS entre os dois grupos. Na análise estatística multivariada pôde-se observar correspondência entre escore corporal e pressão arterial sistólica, sendo que animais com escore corporal acima do ideal apresentaram maiores valores de PAS, sobretudo com o método Doppler ultrassônico (Fig.1).

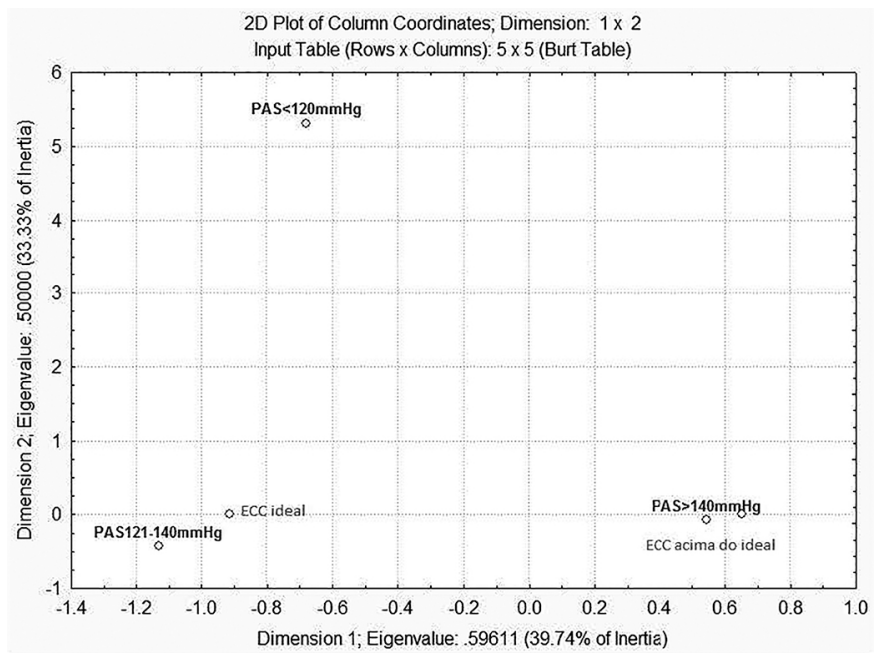

Fig.1 Mapa perceptual gerado pela análise de correspondência múltipla. PAS=pressão arterial sistólica; ECCideal=escore de condição corporal ideal; ECC acima do ideal=escore de condição corporal acima do ideal (escores 8 a 9).

\section{DISCUSSÃO}

O diagnóstico da obesidade realizado por meio da escala de escore corporal é um método prático extensamente utilizado no exame clínico de cães e possui alta repetibilidade, principalmente se realizado por observadores experientes 
e de forma pontual. A desvantagem desse método é não diferenciar o escore corporal em animais com perda de massa magra e ganho de tecido adiposo (Laflamme et al. 1997, Zoran 2010). Desta forma, considerou-se um bom método de avaliação corporal para classificação da obesidade nos animais do presente estudo, uma vez que foi realizado em um único momento em dois grupos distintos, sem avaliar a perda de peso no mesmo animal.

Todos os valores da pressão arterial sistólica encontram-se dentro do intervalo de referência para a espécie e foram semelhantes aos descritos previamente por outros autores, situando-se entre $110 \mathrm{mmHg}$ a $160 \mathrm{mmHg}$ (Stepien et al. 2003, Brown et al. 2007, Pereira Neto et al. 2010). Ao se comparar os valores obtidos pelos dois métodos indiretos de mensuração da PAS, encontrou-se valores inferiores significativos pelo método oscilométrico em cães obesos. Esta informação pode ser reforçada pelos achados da análise estatística multivariada, que demonstraram maior correspondência entre as variáveis ECC acima do ideal, maiores valores de PAS e método doppler ultrassônico. A pressão arterial sistêmica obtida pelo método doppler também foi considerada mais precisa que o método oscilométrico, comparada com a pressão arterial invasiva em estudo com gatos hígidos anestesiados (Petric et al. 2010). Sabe-se que a avaliação da pressão arterial invasiva seria o padrão para a obtenção mais precisa dos valores da PAS e correlação com os métodos indiretos, limitação deste estudo, por ser um método que requer anestesia, procedimento este impossibilitado por se tratar de cães de proprietários da rotina ambulatorial.

Uma hipótese a ser ressaltada é que cães obesos podem apresentar maior depósito de tecido adiposo subcutâneo no membro torácico utilizado, interferindo na captação do pulso arterial e, consequentemente a detecção de falsos valores. No entanto, outros estudos devem ser conduzidos para melhor esclarecer essa suspeita. Igualmente ao observado no grupo controle do presente trabalho, um estudo realizado em cães com peso corporal ideal não verificou diferença significativa ao comparar os dois métodos indiretos (Stepien \& Rappaport 1999).

Segundo Brown et al. (2007), considera-se hipertenso o animal com valores iguais ou superiores a $150 \mathrm{mmHg}$ para a PAS, no entanto, somente uma única mensuração não é o suficiente para estabelecer o diagnóstico, sugerindo se realizar novas medidas, investigar outras doenças de base que possam predispor hipertensão, monitorar por vários dias caso não se encontre a causa primária, além de considerar o efeito do jaleco branco, ou seja, o estresse e ansiedade que os animais apresentam durante o exame em clínicas e hospitais veterinários. Portanto, o discreto aumento da PAS, obtida pelo método doppler nos cães obesos neste estudo, pode ser influência do estresse durante a contenção, mesmo com a adaptação ao ambiente antes da mensuração, uma vez que foi descartado a presença de doença concomitantes, ou corroborar aos achados de Bodey \& Michell (1996), os quais conduziram grande estudo avaliando a influencia de vários fatores como a condição corporal na pressão arterial sistêmica e concluíram que o sobrepeso exerce discreto efeito para a elevação da pressão arterial.
Ao contrario, Montoya e colaboradores (2006) demonstraram a influência da condição corporal sobre a pressão arterial sistêmica e os seus resultados sugeriram correlação positiva entre o escore de condição corporal de nove categorias e a hipertensão nos cães com sobrepeso.

Ao se analisar os valores obtidos de cada cão obeso isoladamente observou-se que $28 \%$ dos animais apresentaram PAS elevada pelo método de Doppler, enquanto que pelo oscilométrico apresentavam valores dentro da normalidade. Chalifoux et al. (1985) demonstraram que hipertensão sistêmica pode ser melhor detectada pelo método doppler em cães não obesos. 0 doppler ultrassônico é um método indireto de baixo custo e confiável para a mensuração da PAS em cães obesos, podendo ser utilizado facilmente na prática clínica, já que o método direto é mais invasivo para ser realizado rotineiramente em cães de proprietário. No entanto, o diagnóstico de hipertensão deve ser cauteloso, pois não deve ser realizado apenas por meio de simples aferição da pressão arterial sistólica e sim, associado aos sinais clínicos, presença de fatores predisponentes e confirmado por mensurações repetidas ao longo do tempo.

A presença e a intensidade das alterações cardiovasculares em pacientes obesos dependem e são proporcionais ao tempo de instalação e convivência com as alterações hemodinâmicas e gravidade da obesidade (Rocha et al. 2007). Portanto, a ausência da elevação significativa da pressão arterial verificada nessa amostra de cães obesos por meio de diferentes métodos de mensuração indireta, pode ser devido ao fato de alguns animais avaliados não apresentarem obesidade pronunciada (escore de condição corporal $=9$ ) ou pelo fato dos cães não possuírem expectativa de vida longa o suficiente em excesso de peso corporal, condição necessária para afetar o controle da pressão arterial sistêmica.

\section{CONCLUSÕES}

Os resultados do presente trabalho demonstram que método doppler ultrassônico para mensuração indireta da pressão arterial gera valores mais elevados da PAS comparado ao método oscilométrico em cães obesos, desta forma, sugere-se considerar essas variações da metodologia empregada no diagnóstico de alterações da pressão arterial em cães com excesso de peso.

Concluiu-se também que cães obesos apresentam pressão arterial sistólica dentro dos valores do intervalo de referência para a espécie.

Agradecimentos.- Os autores agradecem a Capes, pela concessão de bolsa de doutorado à primeira autora; Mogiana Alimentos (Guabi) pelo suporte financeiro ao Serviço de Nutrição Clínica de Cães e Gatos do Hospital Veterinário da FCAV/Unesp e a todos os proprietários dos animais que aceitaram gentilmente participar deste estudo.

\section{REFERÊNCIAS}

Bodey A.R., Young L.E., Bartram D.H., Diamond M.J. \& Michell A.R. 1994. A comparison of direct and indirect (oscillometric) measurements of arterial blood pressure in anaesthetized dogs, using tail and limbs cuffs. Res. Vet. Sci. 57:265-269.

Bodey A.R., Michell A.R., Bovee K.C., Buranakuri C. \& Garg T. 1996. Comparison of direct and indirect (oscillometric) measurement of arterial blood pressure in conscious dog. Res. Vet. Sci. 61:17-21. 
Bodey A.R. \& Michell A.R. 1996. Epidemiological study of blood pressure in domestic dogs. J. Small Anim. Pract. 37:16-125.

Brown S.A. \& Henik R.A. 1998. Diagnosis and treatment of systemic hypertension. J. Small Anim. Pract. 28(6):1481-1494.

Brown S., Atkins C., Bagley R., Carr A., Cowgill L., Davidson M., Egner B., Elliot J., Henik R., Labato M., Littman M., Polzin D., Ross L., Snyder P. \& Stepien R. 2007. Guidelines for the identification, evaluation, and management of systemic hypertesion in dogs and cats. J. Vet. Intern. Med. 21:542-558.

Carr A. 2001. Measuring blood pressure in dogs and cats. Vet. Med. 2:135144.

Chalifoux A., Dallaire A., Blaias D., Laviriére N. \& Pelletier N. 1985. Evaluation of the arterial blood pressure of dogs by two noninvasive methods. Can. J. Comp. Med. 49:419-423.

Hair J.F., Black W.C., Babin B.J. \& Anderson R.E. 2006. Multivariate Data Analysis. $6^{\text {th }}$ ed. Hardcover, New York, 899p.

Laflamme D.P., Kuhlman G. \& Lawler D.F. 1997. Evaluation of weight loss protocols for dogs. J. Am. Anim. Hosp. Assoc. 33:253-259.

Laflamme D.P. 2001. Challenges with weight-reduction studies. Comp. Cont. Educ. Pract. Vet. 23:45-50.

Laflamme D.P. 2006. Understanding and managing obesity in dogs and cats. J. Small Anim. Pract. 36:1283-1295.

Meurs K.M., Miller M.W. \& Slater M.R. 1996. Comparison of the indirect oscillometric and direct arterial methods for blood pressure measurement in anaesthetized dogs. J. Am. Anim. Hosp. Assoc. 32:471-475.

Montoya J.A., Morris P.J., Bautista I., Candelaria Juste M., Suarez L., Peña C.,
Hackett R.M. \& Rawlings J. 2006. Hypertension: a risk factor associated with weight status in dogs. J. Nutr. 136:2011S-2013S.

Pereira Neto G.B., Brunetto M.A., Carciofi A.C. \& Camacho A.A. 2010. Effects of weight loss on the cardiac parameters of obese dogs. Pesq. Vet. Bras. 30(2):167-171.

Petric A.D., Petra Z., Jerneja S. \& Alenka S. 2010. Comparison of high definition oscillometric an Doppler ultrasonic devices for measuring blood pressure in anaesthetised cats. J. Feline Med. Surg. 12(10): 731-737.

Remillard R.L., Ross J.N. \& Eddy J.B. 1991. Variance of indirect blood pressure measurements and prevalence of hypertension in clinically normal dogs. Am. J. Vet. Res. 52:561-565.

Rocha I.E.G.M., Victor E.G., Braga M.C., Barbosa e Silva O. \& Becker M.M.C. 2007. Avaliação ecocardiográfica em obesos assintomáticos. Arq. Bras. Cardiol. 88(1):52-58.

Schlotzhauer S. \& Littel R.C. 1997. SAS system for elementary statistical analysis. $2^{\text {nd }}$ ed. SAS Institute, Cary, NC, p.183-269.

Stepien R.L. \& Rapoport G.S. 1999. Clinical comparison of three methods to measured blood pressure in non sedated dogs. J. Am. Hosp. Assoc. 215:1623-1628.

Stepien R.L., Rapoport G.S., Henik R.A., Wenholz L. \& Thomaz C.B. 2003. Comparative diagnostic test characteristics of oscillometric and doppler ultrasonographic methods detection of systolic hypertension in dogs. J. Vet. Int. Med. 17:65-72.

Zoran D.L. 2010. Obesity in dogs and cats: a metabolic and endocrine disorder. J. Small Anim. Pract. 40:221-239. 